\title{
EDTA-functionalized $\mathrm{Fe}_{3} \mathrm{O}_{4}$ nanoparticles
}

\author{
A.G. Magdalena $^{\text {a, }}{ }^{*}$, I.M.B. Silva ${ }^{\text {a }}$, R.F.C. Marques ${ }^{\text {b }}$, A.R.F. Pipi ${ }^{\text {c }}$, P.N. Lisboa-Filho ${ }^{\text {, }}$, \\ M. Jafelicci Jr. ${ }^{\mathrm{b}}$ \\ a Department of Chemistry, School of Sciences, São Paulo State University (UNESP), Campus of Bauru, Bauru, São Paulo, Brazil \\ ${ }^{\mathrm{b}}$ Department of Physical and Chemistry, Institute of Chemistry, São Paulo State University (UNESP), Campus of Araraquara, Araraquara, São Paulo, Brazil

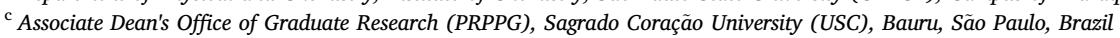 \\ ${ }^{\mathrm{d}}$ Department of Physics, School of Sciences, São Paulo State University (UNESP), Campus of Bauru, Bauru, São Paulo, Brazil
}

\section{A R T I C L E I N F O}

\section{Keywords:}

Magnetite

EDTA

Magnetic nanoparticles

Functionalization

\begin{abstract}
A B S T R A C T
This study analyzes the synthesis and characterization of functionalized $\mathrm{Fe}_{3} \mathrm{O}_{4}$ nanoparticles by ethylenediaminetetraacetic acid (EDTA). The syntheses were performed using the co-precipitation method under different experimental conditions: nitrogen atmosphere versus ambient atmospheric conditions, and temperatures of $25^{\circ} \mathrm{C}$ versus $90^{\circ} \mathrm{C}$. X-ray diffraction techniques, Fourier transform infrared (FTIR) spectroscopy, zeta potential, and transmission electron microscopy (TEM) were used to characterize these nanoparticles. The co-precipitation method produced high homogeneity in nanostructure shape and size. The functionalization of the magnetite surface was confirmed by the FTIR analyses and the development of new bands associated with EDTA as well as by zeta potential change. The addition of EDTA was also found to change the mechanism of nucleation and nanostructure growth; EDTA was found to favor nucleation, thus decreasing nanoparticle size.
\end{abstract}

\section{Introduction}

Iron oxide nanoparticles are the most commonly used today types of nanostructured system, which exhibit magnetic properties. Iron oxide nanoparticles have also been used in hyperthermia and theranostic devices, a usage which has increased substantially in the last ten years [1-3].

The most frequently studied nanoparticles are iron-based nanoparticles, which are known as nanoscaled zero valent iron (nZVI), maghemite nanoparticles $\left(\gamma-\mathrm{Fe}_{2} \mathrm{O}_{3}\right)$ and magnetite nanoparticles $\left(\mathrm{Fe}_{3} \mathrm{O}_{4}\right)$ due to their proven biocompatibilities $[1,2,4-6]$. These iron-based nanoparticles exhibit unique chemical properties resulting from different iron oxidation states. They are widely used as catalysts in environmental remediation, and as drug delivery vehicles in biomedicine [7]. Interest in nanoparticles has increased in part because they are simple to prepare and are chemically stable when stored in colloidal suspension form. Colloidal suspensions of stabilized magnetic nanoparticles are referred to as ferrofluids and may interact with a external magnetic field [8]. $\mathrm{Fe}_{3} \mathrm{O}_{4}$ nanoparticles possess the ability to adsorb heavy metals in an aqueous medium through physical and chemical interactions with these heavy metals [7]. However, the major mechanism of contaminant removal from an aqueous medium that employs $\gamma-\mathrm{Fe}_{2} \mathrm{O}_{3}$ nanoparticles is physical adsorption [7].

One inevitable problem associated with these particles in this size range is their long-term instability. These particles tend to pursue agglomerates to reduce the energy associated with the increased surfacearea-to-volume ratio of nanoparticles. Furthermore, the nanoparticles are chemically very active and easily oxidizes in the air, which generally results in low magnetic properties and dispersibility. For many applications, it is crucial to develop chemical and colloidal protection strategies to chemically stabilize magnetic nanoparticles against degradation during and after synthesis as well as colloidal stability through electrostatical and/or stericstabilization $[9,10]$. These strategies include a coverage graft with organic molecules (surfactants or polymers) or coverage with an inorganic film (silica or carbon). It is important to note that, in many cases of protection, the shell does not only stabilize the nanoparticles: it may also be used for further functionalities (such as with other nanoparticles or different ligands, depending on the desired application) $[1,11]$. Along this vein, recent studies [12-14] have demonstrated the high affinity of ethylenediaminetetraacetic acid (EDTA) for iron oxide nanoparticles, which may improve colloidal dispersibility, decrease high surface energy, and consequently decrease the nanoparticles agglomeration.

The chelating potential of EDTA may be used to synthesize EDTA-

\footnotetext{
* Corresponding author.

E-mail address: aroldogm@fc.unesp.br (A.G. Magdalena).
} 
Table 1

Nomenclature of samples.

\begin{tabular}{|c|c|c|c|}
\hline Sample & Name & Sample & Name \\
\hline $\mathrm{Fe}_{3} \mathrm{O}_{4} 25^{\circ} \mathrm{C}$; $\mathrm{AMB}$ & NP25AMB & $\begin{array}{l}\mathrm{Fe}_{3} \mathrm{O}_{4} \text {-EDTA- } \\
0.002 \\
\text { M } 25^{\circ} \mathrm{C} \text {; AMB. }\end{array}$ & NP-L0.002M25AMB \\
\hline $\mathrm{Fe}_{3} \mathrm{O}_{4} 25^{\circ} \mathrm{C} ; \mathrm{N}_{2}$ & NP25N2 & $\begin{array}{l}\mathrm{Fe}_{3} \mathrm{O}_{4} \text {-EDTA- } \\
0.002 \\
\mathrm{M} 25^{\circ} \mathrm{C} ; \mathrm{N}_{2}\end{array}$ & NP-L0.002M25N2 \\
\hline $\mathrm{Fe}_{3} \mathrm{O}_{4} 90^{\circ} \mathrm{C}$; $\mathrm{AMB}$ & NP90AMB & $\begin{array}{l}\mathrm{Fe}_{3} \mathrm{O}_{4} \text {-EDTA- } \\
0.002 \\
\mathrm{M} 90{ }^{\circ} \mathrm{C} ; \mathrm{AMB} .\end{array}$ & NP-L0.002M90AMB \\
\hline $\mathrm{Fe}_{3} \mathrm{O}_{4} 90^{\circ} \mathrm{C} ; \mathrm{N}_{2}$ & NP90N2 & $\begin{array}{l}\mathrm{Fe}_{3} \mathrm{O}_{4} \text {-EDTA- } \\
0.002 \\
\mathrm{M} 90{ }^{\circ} \mathrm{C} ; \mathrm{N}_{2} \\
\mathrm{Fe}_{3} \mathrm{O}_{4} \text {-EDTA } 0.02 \\
\mathrm{M} 90{ }^{\circ} \mathrm{C} ; \mathrm{N}_{2} \\
\mathrm{Fe}_{3} \mathrm{O}_{4} \text {-EDTA } 0.2 \\
\mathrm{M} 90^{\circ} \mathrm{C} ; \mathrm{N}_{2}\end{array}$ & $\begin{array}{l}\text { NP-L0.002M90N2 } \\
\text { NP-L0.02M90N2 } \\
\text { NP-L0.2M90N2 }\end{array}$ \\
\hline
\end{tabular}

functionalized $\mathrm{Fe}_{3} \mathrm{O}_{4}$ nanoparticles, which may be proved to be promising for studies on the adsorption of heavy metals due to the EDTA chelating cations, thus forming complexes with their structures $[12,13]$.

The present contribution presents a study in the synthesis conditions and characterization of EDTA-functionalized $\mathrm{Fe}_{3} \mathrm{O}_{4}$ nanoparticles using the co-precipitation method of synthesis and changing the experimental conditions in order to carefully determine the importance of the experimental parameters in the synthesis of these compounds. The considered parameters were synthesis temperature $\left(25{ }^{\circ} \mathrm{C}\right.$ versus $\left.90{ }^{\circ} \mathrm{C}\right)$, atmospheric conditions (presence versus absence of $\mathrm{N}_{2}$ ), and EDTA concentration $\left(0.2,0.02\right.$, and $\left.0.002 \mathrm{~mol} \mathrm{~L}^{-1}\right)$.

\section{Experimental}

The chemical reagents used for the synthesis of EDTA-functionalized $\mathrm{Fe}_{3} \mathrm{O}_{4}$ nanoparticles were iron (II) chloride tetrahydrate (Merck), iron (III) chloride hexahydrate (Aldrich), ammonium hydroxide $\sim 28 \%$ (Synth), and EDTA (Synth).

The nanomaterials were synthesized by the co-precipitation method with some modifications $[1,8,10,12,13]$. Four syntheses were performed in the case of the $\mathrm{Fe}_{3} \mathrm{O}_{4}$ nanoparticles and another four ones were performed in the case of the $\mathrm{Fe}_{3} \mathrm{O}_{4}$-EDTA particles under the following four experimental conditions: (1) controlled synthesis temperature at $25{ }^{\circ} \mathrm{C}$ without inert atmosphere; (2) controlled synthesis temperature at $25^{\circ} \mathrm{C}$ under nitrogen atmosphere; (3) controlled synthesis temperature at $90^{\circ} \mathrm{C}$ under reflux and without inert atmosphere; and (4) controlled synthesis

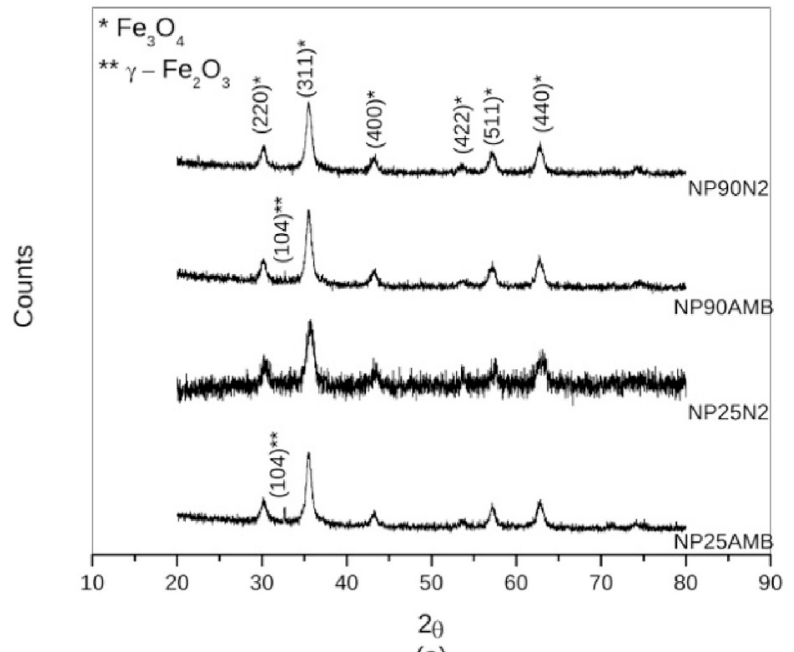

temperature at $90{ }^{\circ} \mathrm{C}$ under reflux and nitrogen atmosphere.

In the first step, Fe3O4 nanoparticles were synthesized using $100 \mathrm{~mL}$ of precursor solution containing $0.030 \mathrm{~mol}$ iron (III) chloride hexahydrate and $0.015 \mathrm{~mol}$ iron (II) chloride tetrahydrate, to which it was added $10 \mathrm{~mL}$ of ammonium hydroxide. This mixture was left under constant agitation for $1 \mathrm{~h}$ at the experimental temperature and atmospheric conditions in (1) to (4). The final precipitate was separated using a magnet and washed many times with distilled water and acetone.

In the second step, the EDTA-functionalized $\mathrm{Fe}_{3} \mathrm{O}_{4}$ nanoparticles were synthesized by using the same procedure used for the synthesis of the $\mathrm{Fe}_{3} \mathrm{O}_{4}$ nanoparticles. Then, it was added of $50 \mathrm{~mL}$ of a $0.002 \mathrm{~mol} \mathrm{~L}^{-1}$ EDTA solution soon after the ammonium hydroxide was added. Two additional $\mathrm{Fe}_{3} \mathrm{O}_{4}$-EDTA nanoparticle syntheses were performed using the experimental conditions in (4) in a similar procedure, but changing the EDTA concentration. In these changes, $50 \mathrm{~mL}$ solutions with $0.02 \mathrm{~mol} \mathrm{~L}^{-1}$ of EDTA or $0.2 \mathrm{~mol} \mathrm{~L}^{-1}$ of EDTA were used.

In Table 1 are presented the different synthesis prepared with the parameters evaluated. As an example, the sample NP25N2 and NPL2mM25AMB represents $\mathrm{NP}-\mathrm{Fe}_{3} \mathrm{O}_{4}$ nanoparticles; L - EDTA ligand; $0.002 \mathrm{~mol} \mathrm{~L}^{-1}, 0.02 \mathrm{~mol} \mathrm{~L}^{-1}$ or $0.2 \mathrm{~mol} \mathrm{~L}^{-1}$ - EDTA concentration; 25 or 90 - synthesis temperature in ${ }^{\circ} \mathrm{C}$, and $\mathrm{N}_{2}$ or AMB - atmospheric conditions (nitrogen atmosphere or without inert atmosphere conditions, respectively).

Nanoparticles samples were characterized based on the X-ray diffractometer patterns obtained from a Rigaku-Rint 2000 diffractometer with $\mathrm{CuK}_{\alpha}$ radiation. Transmission electron microscopy (TEM) was performed using a Philips CM-200 instrument with a super twin a-lens. The FTIR spectrum was measured using a Vertex 70 spectrometer (Bruker instruments). The Zetasizer Nano ZS system (Malvern Instruments) was also used in the zeta potential measurements. Variations in $\mathrm{pH}$ were controlled using $\mathrm{HCl} / \mathrm{NaOH}$.

\section{Results and discussion}

Fig. 1 shows the $\mathrm{X}$-ray diffractograms from different synthesis parameters for the $\mathrm{Fe}_{3} \mathrm{O}_{4}$ nanoparticles (Fig. 1-a) and the $\mathrm{Fe}_{3} \mathrm{O}_{4}$-EDTA nanoparticles (Fig. 1-b). These X-ray diffractograms show the crystallographic planes representing the diffraction of magnetite in all of the systems studied. The syntheses under ambient atmospheric conditions (NP25AMB, NO90AMB, and NP-L0.002M25AMB) produced a crystallographic plane representing magnetite $\left(\mathrm{Fe}_{3} \mathrm{O}_{4}\right)$ and maghemite $\left(\gamma-\mathrm{Fe}_{2} \mathrm{O}_{3}\right)$, a finding that indicates oxidation during synthesis. According to Gupta et al. [1], organic or inorganic molecules are typically used during the precipitation process to inhibit the oxidation of nanoparticles. The

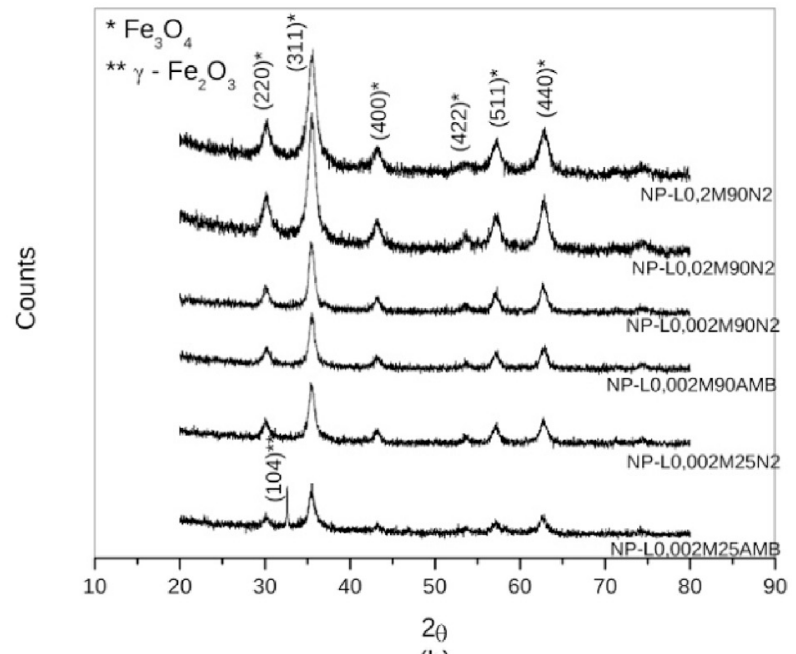

(b)

Fig. 1. X-ray diffraction patterns obtained for the samples. The magnetite (JCPDS card no. 89-0688) and maghemite (JCPDS card no. 89-0597) patterns from the JCPDS database. 


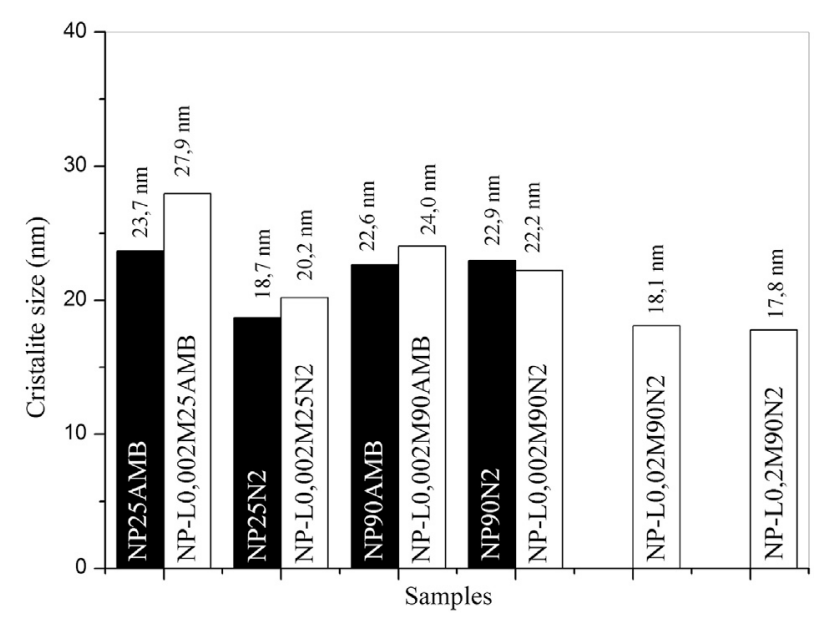

Fig. 2. Crystallite size obtained from the Scherrer equation to the samples.

magnetite (JCPDS card no. 89-0688) and maghemite (JCPDS card no. 89-0597) patterns from the JCPDS database for were included for comparison.

Even so, the oxidation of NP-L0.002M25AMB was found to occur in this experiment. However, in the case of NP-L0.002M90AMB and the other nanoparticles, the interaction was made by chemical binding between magnetite and EDTA molecules during synthesis. The EDTA molecules are attached in the nanoparticles. The EDTA-functionalized nanoparticles inhibits oxidation process of magnetite $\left(\mathrm{Fe}_{3} \mathrm{O}_{4}\right)$ leading to maghemite $\left(\gamma-\mathrm{Fe}_{2} \mathrm{O}_{3}\right)$. Kinetically controlled oxidation of ferrous species may be decreased through the use of a system to remove oxygen from the reaction medium. This may be obtained from the gas nitrogen streaming during the synthesis. The ideal molar reaction for the synthesis of magnetite is $2: 1$ ratio to $\mathrm{Fe}^{3+}: \mathrm{Fe}^{2+}$. To guarantee this ratio, inert atmosphere is used in the reaction medium. This control is very important: it may modify the surface properties of these materials, as well as the magnetic properties, which tend to decrease with oxidation.

As can be seen, temperature does not seem to significantly influence the stability of the phases. Iron oxide nanoparticles exhibited greater crystallinity in the samples synthesized at $90{ }^{\circ} \mathrm{C}$ than in those performed at $25{ }^{\circ} \mathrm{C}$. Taking into account that for these systems, nucleation must occur before the growth of pre-formed nucleus, the temperature should favor the growth stage of the samples synthesized at $25{ }^{\circ} \mathrm{C}$, thus increasing the diffusion of iron atoms to the nucleus that is formed. At this point, the dominant stage begins to occur as a growth mechanism, meaning that the speed of iron atom aggregation in the nanoparticles comes to depend on the quantity of these atoms around the nucleus.

The graph in Fig. 2 shows an estimate of the crystalline sizes obtained using the Scherrer equation [15]. In this work, the Scherrer's equation was used as the first approximation to determine the particle size, taking in consideration the uncertainty associated to this equation, as previously discussed by Tomaszewski [16]. Further up, the particles size were determined using the images obtained by TEM (Figs. 3-b, 4-b and 5), which is more appropriate to this purpose. The result indicates that all of the synthesized materials exhibit a nanometric particle size between 17.8 and $27.9 \mathrm{~nm}$. The EDTA-functionalized $\mathrm{Fe}_{3} \mathrm{O}_{4}$ samples also exhibited smaller particle sizes. The use of nitrogen atmosphere prevented the oxidation process and aided in the control of particle size. All of the samples to which nitrogen gas streaming was applied were smaller than those in which ambient atmospheric conditions were used. This effect was found to be more pronounced at $25^{\circ} \mathrm{C}$. Because the growth process is favored at $90{ }^{\circ} \mathrm{C}$, the crystallite sizes are very similar, a result that indicates that the expected findings involving temperature are consistent with the experimental data.

Fig. 2 also shows that the samplesNP-L0.002M90N2, NP-
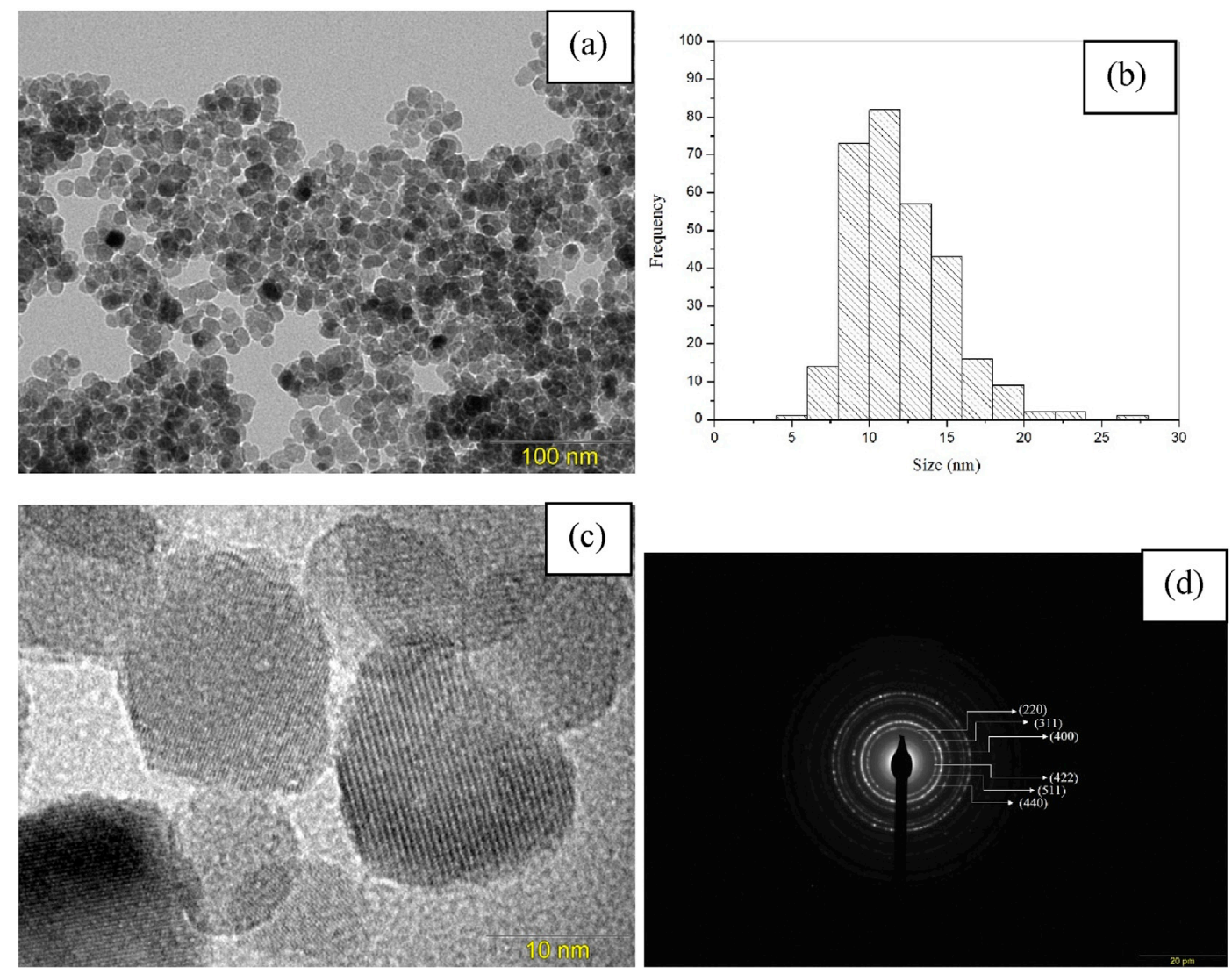

Fig. 3. Transmission electron microscopy for the nanoparticle referred to as NP90N2. 

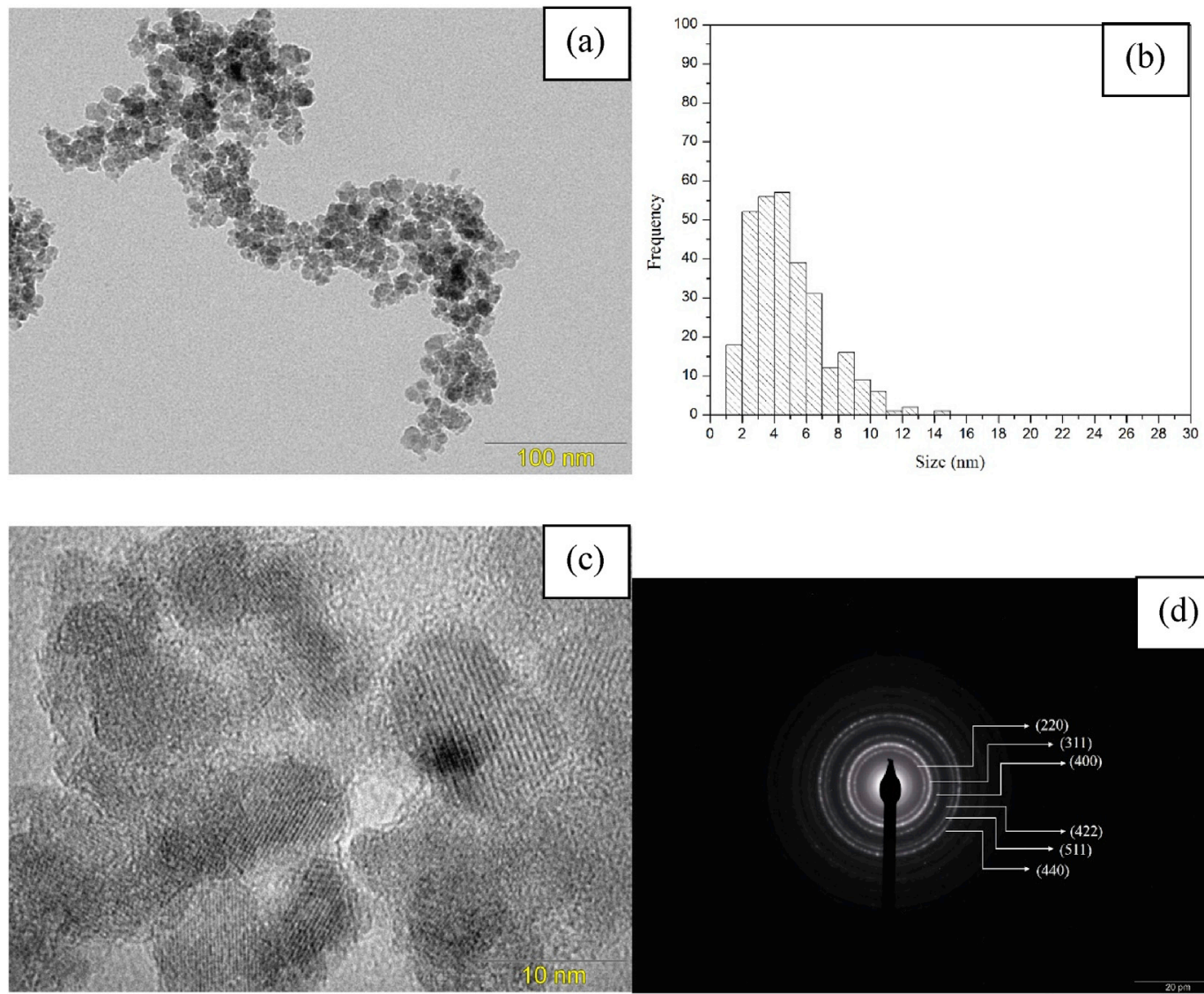

c)

Fig. 4. Transmission electron microscopy for the nanoparticle referred to as NP-L0.2M90N2

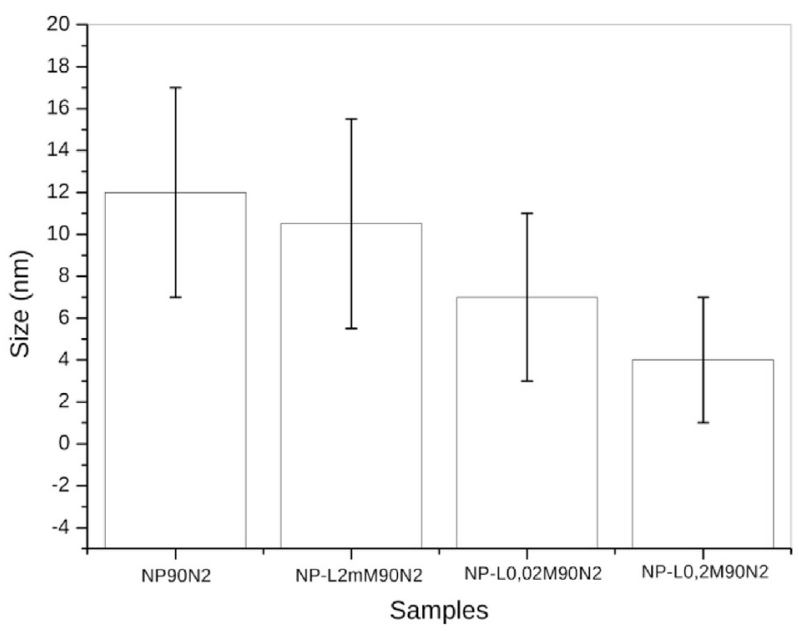

Fig. 5. Average size of some of the synthesized nanostructures.

L0.02M90N2, and NP-L0.2M90N2 nanostructures were found to be smaller in size than all of the other structures studied. As its concentrations increase, EDTA likely changes the mechanism of nanoparticle formation, which in turn, favors more nucleation than in the growth stage. In this case, EDTA is likely functioning as a barrier to the nuclei that are formed, resulting in decreased nanoparticle size.

The electron transmission micrographs in Figs. 3 and 4 shows that the nanostructures are highly homogeneous in shape and size. The micrographs (Fig. 3 and c) show that the average nanoparticle size was found to be approximately $12 \mathrm{~nm}$ in the case of NP90N2 nanoparticle, as verified in the histogram in Fig. 3-b. Similarly, the micrographs in Fig. 4a and c reflect the average size of $4 \mathrm{~nm}$ for the case of the NP-L0.2M90N2 nanoparticle, as presented in the histogram in Fig. 4c.

The transmission electron micrographs clearly show the crystallographic planes present in the nanostructures in Figs. $3 c$ and $4 c$, and Figs. $3 \mathrm{~d}$ and $4 \mathrm{~d}$ represent the electron diffraction in these nanostructures. These results are consistent with the crystallographic planes presented in the X-ray diffraction patterns shown in Fig. 1.

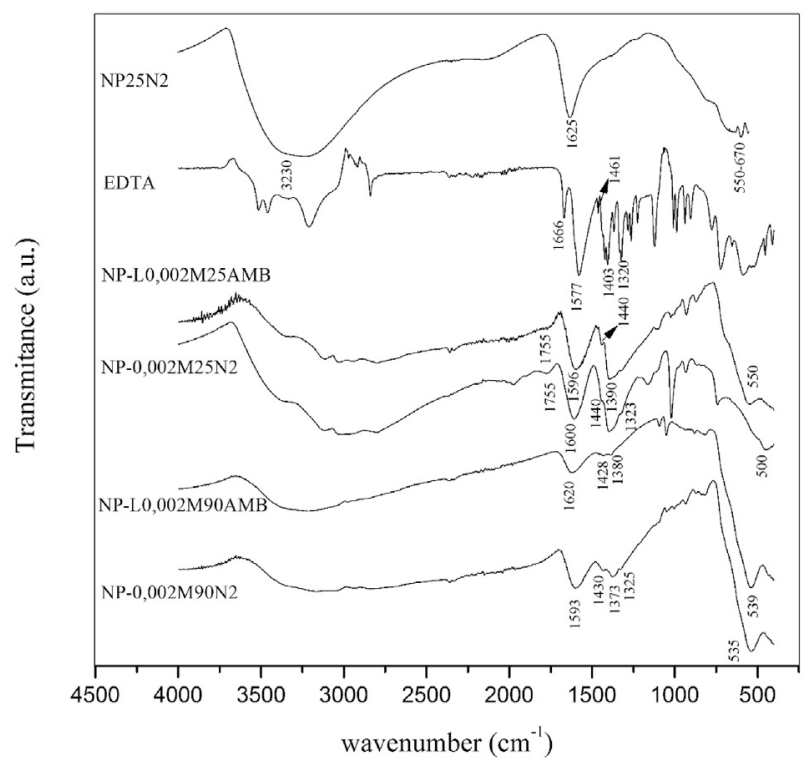

Fig. 6. Infrared spectra from investigated functionalized iron oxide nanoparticles. The main bands are featured. 
Table 2

Description of the FTIR bands assignment of the spectra shown in Fig. 6.

\begin{tabular}{|c|c|c|c|c|c|}
\hline NP25N2 & EDTA & NP-L0.002M25AMB & NP-L0.002M25N2 & NP-L0.002M90AMB & NP-L0.002M90N2 \\
\hline$(550-670) \mathrm{Fe}-\mathrm{O}$ & - & $(550)-\mathrm{Fe}-\mathrm{O}$ & $(500)-\mathrm{Fe}-\mathrm{O}$ & $(539)-\mathrm{Fe}-\mathrm{O}$ & $(535)-\mathrm{Fe}-\mathrm{O}$ \\
\hline- & $(900-960) \cup(C-C)$ & $(870-930) \cup(C-C)$ & $(933) \cup(\mathrm{C}-\mathrm{C})$ & $(880) \cup(C-C)$ & $(870-930) \cup(C-C)$ \\
\hline- & $(1006)-\mathrm{v}\left[\mathrm{N}\left(-\mathrm{CH}_{2}-\right)_{3}\right]$ & $\begin{array}{l}(1020.1006 \text { and } 972) v \\
{\left[\mathrm{~N}\left(-\mathrm{CH}_{2}-\right)_{3}\right]}\end{array}$ & $\begin{array}{l}(1018 \text { and } 975) \mathrm{v} \\
{\left[\mathrm{N}\left(-\mathrm{CH}_{2}-\right)_{3}\right]}\end{array}$ & $(1091.1048$ and 975$) \cup\left[\mathrm{N}\left(-\mathrm{CH}_{2}-\right)_{3}\right]$ & $(1044-1002)$ v $\left[\mathrm{N}\left(-\mathrm{CH}_{2}-\right)_{3}\right]$ \\
\hline- & $(1320) \delta\left(\mathrm{NH}^{+}\right)$ & $(1325) \delta\left(\mathrm{NH}^{+}\right)$ & $(1323) \delta\left(\mathrm{NH}^{+}\right)$ & - & $(1325) \delta\left(\mathrm{NH}^{+}\right)$ \\
\hline- & $(1403) \cup(\mathrm{C}-\mathrm{O}) \mathrm{COOH}$ & $(1390) \cup(\mathrm{C}-\mathrm{O}) \mathrm{COOH}$ & $(1390) \cup(\mathrm{C}-\mathrm{O}) \mathrm{COOH}$ & $(1382) \cup(\mathrm{C}-\mathrm{O}) \mathrm{COOH}$ & $(1373)$ v $(\mathrm{C}-\mathrm{O}) \mathrm{COOH}$ \\
\hline- & $(1461) v_{\mathrm{si}}\left(\mathrm{COO}^{-}\right)$ & $(1440) v_{\mathrm{si}}\left(\mathrm{COO}^{-}\right)$ & $(1440) v_{\mathrm{si}}\left(\mathrm{COO}^{-}\right)$ & $(1428) v_{\mathrm{si}}\left(\mathrm{COO}^{-}\right)$ & $(1430) v_{\mathrm{si}}\left(\mathrm{COO}^{-}\right)$ \\
\hline- & $(1577) v_{\text {ass }}\left(\mathrm{COO}^{-}\right)$ & $(1596) v_{\text {ass }}\left(\mathrm{COO}^{-}\right)$ & $(1600) v_{\text {ass }}\left(\mathrm{COO}^{-}\right)$ & $(1620) v_{\text {ass }}\left(\mathrm{COO}^{-}\right)$ & $(1593) v_{\text {ass }}\left(\mathrm{COO}^{-}\right)$ \\
\hline$(1625) \cup\left(\mathrm{H}_{2} \mathrm{O}\right)$ & - & - & - & - & - \\
\hline- & $(1666) \cup(\mathrm{C}=\mathrm{O}) \mathrm{COOH}$ & $(1755) \cup(\mathrm{C}=\mathrm{O}) \mathrm{COOH}$ & $(1774) \cup(\mathrm{C}=\mathrm{O}) \mathrm{COOH}$ & - & - \\
\hline- & $(2837-3210) \cup(\mathrm{C}-\mathrm{H})$ & $(2800-3112) \cup(\mathrm{C}-\mathrm{H})$ & $(2792-3119) \cup(\mathrm{C}-\mathrm{H})$ & - & $(2844-3042) \cup(\mathrm{C}-\mathrm{H})$ \\
\hline$(3230) \cup(\mathrm{O}-\mathrm{H})$ & $(3458-3512) \cup(\mathrm{O}-\mathrm{H})$ & $(3340) \cup(\mathrm{O}-\mathrm{H})$ & $(3362) \cup(\mathrm{O}-\mathrm{H})$ & $(3221) \cup(\mathrm{O}-\mathrm{H})$ & $(3200) \cup(\mathrm{O}-\mathrm{H})$ \\
\hline
\end{tabular}

The XRD and TEM results are in agreement with the reported data by Refs. [10,17]. Because in the homogeneous nucleation process there should be supersaturation of the precursors, that should be sufficient for the formation of the critical radius for the occurrence of the precipitate. Furthermore, the nanoparticles precipitate obtained shows high surface energy and elevated surface charge and tend to form agglomerates to minimize this surface energy, thus increasing the average size of the nanoparticles. To minimize the high values of surface energy, it commonly adds organic and/or inorganic molecules, which will interact with the nanoparticles to avoid the agglomerate and consequently allows the kinetic control of growth stage.

The graph in Fig. 5 shows the average sizes of the NP90N2, NPL0.002 M 90N2, NP-L0.02M90N2, and NP-L0.2M90N2 nanostructures. The nanoparticle sizes were determined based on the histograms from the TEM analyses. The results confirm the proposal that the addition of EDTA will favor the nucleation stage of the nanoparticles. The graph in Fig. 5 also shows the influence of EDTA concentration on particle size: the results reflect the decrease in nanoparticle size as EDTA concentration increased, which is consistent with the results provided by the use of the X-ray diffraction technique.

In order to confirm the functionalization of EDTA on the surface of the nanoparticles, infrared spectroscopy analyses were performed and the resulting graphs are shown in Fig. 6.

In the infrared spectrum of magnetite (NP25N2) shown in Fig. 6, three bands are visible: the first one is found between 560 and $670 \mathrm{~cm}^{-1}$ and is assigned to the binding of iron to oxygen, a property required for the formation of $\mathrm{Fe}_{3} \mathrm{O}_{4}$; the second band is found near $1625 \mathrm{~cm}^{-1}$ and is the result of the absorption of water molecules; and the third band, found near $3230 \mathrm{~cm}^{-1}$, is associated with stretching $\mathrm{v}(\mathrm{O}-\mathrm{H})$. The latter band proves the formation of hydroxyl groups on the surface of magnetite. Fig. 6 also allows for an analysis of the pure EDTA spectrum, on which a series of bands were found. Bands were found at $900-960 \mathrm{~cm}^{-1}$ due to the $\nu(\mathrm{C}-\mathrm{C})$ axial deformations, at $1006 \mathrm{~cm}^{-1}$ due to the asymmetrical axial deformations in the group $\nu\left[\mathrm{N}\left(-\mathrm{CH}_{2}-\right)_{3}\right]$ of the tertiary amines, at $1320 \mathrm{~cm}^{-1}$, which is attributed to angular deformation $\delta\left(\mathrm{NH}^{+}\right)$, near 1461 and $1577 \mathrm{~cm}^{-1}$, which is attributed to symmetrical and asymmetrical axial deformation $\mathrm{v}\left(\mathrm{COO}^{-}\right)$, and between 1403 and $1666 \mathrm{~cm}^{-1}$, which corresponds to vibrational motions $\nu(\mathrm{C}-\mathrm{O})$ and $\nu(\mathrm{C}=\mathrm{O})$ of the $-\mathrm{COOH}$ group. There were also many bands between 2837 and $3210 \mathrm{~cm}^{-1}$, which were associated with the $\nu(\mathrm{C}-\mathrm{H})$ axial deformities of the $\mathrm{CH}_{2}$ groups. Finally, the bands between 3458 and $3512 \mathrm{~cm}^{-1}$ are attributed to stretching $\mathrm{o}(\mathrm{O}-\mathrm{H})$ resulting from water and hydration in this compound. Table 2 contains all of the bands found for each of the samples analyzed.

Infrared spectra attributed to the functionalization of the EDTA on the surface of $\mathrm{Fe}_{3} \mathrm{O}_{4}$ nanoparticles are the in Fig. 6 (NP-L2mM25AMB, NPL2mM25N2, NP-L2mM90AMB, and NP-L2mM90N2). FTIR spectra for the NP-L0.02M90N2 and NP-L0,2M90N2 samples are very similar to those of the NP-L0.002M90N2 nanoparticles. The appearance of new FTIR bands for pure magnetite can also be seen and they are attributed to the functionalized EDTA molecule on the magnetic nanoparticles surface. Another remarked aspect of these graphs is the decrease in band intensity associated to the stretching $v(\mathrm{O}-\mathrm{H})$ to approximately $3230 \mathrm{~cm}^{-1}$. This result indicates that, in cases of functionalization with EDTA, these groups are recovered and/or are participating in chemical reactions. Another finding is that magnetite may bind with the carboxylates of EDTA, since the carboxylates exhibit considerable distortion in the $\mathrm{v}$ $(\mathrm{C}-\mathrm{O})$ and stretching $\mathrm{v}_{\text {ass }}\left(\mathrm{COO}^{-1}\right)$ bands of pure EDTA, a result which

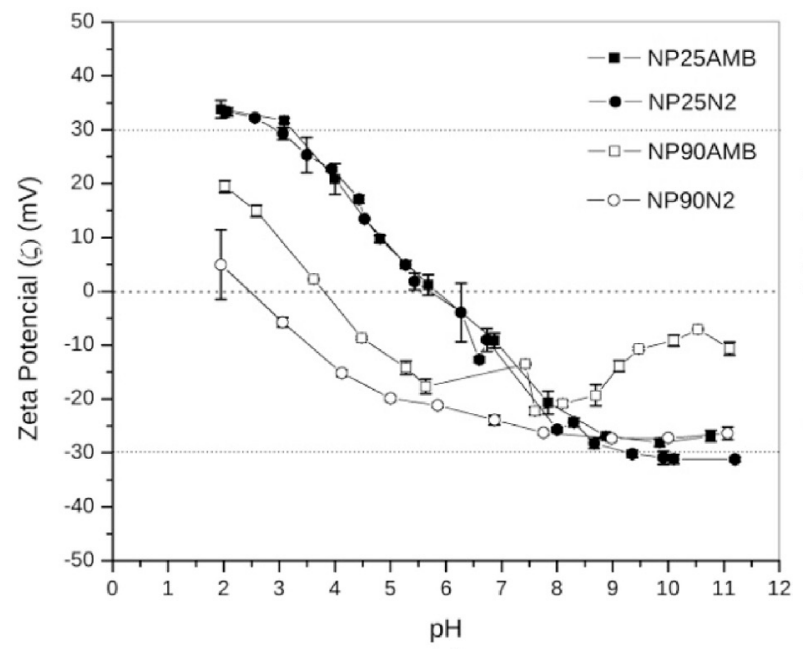

(a)

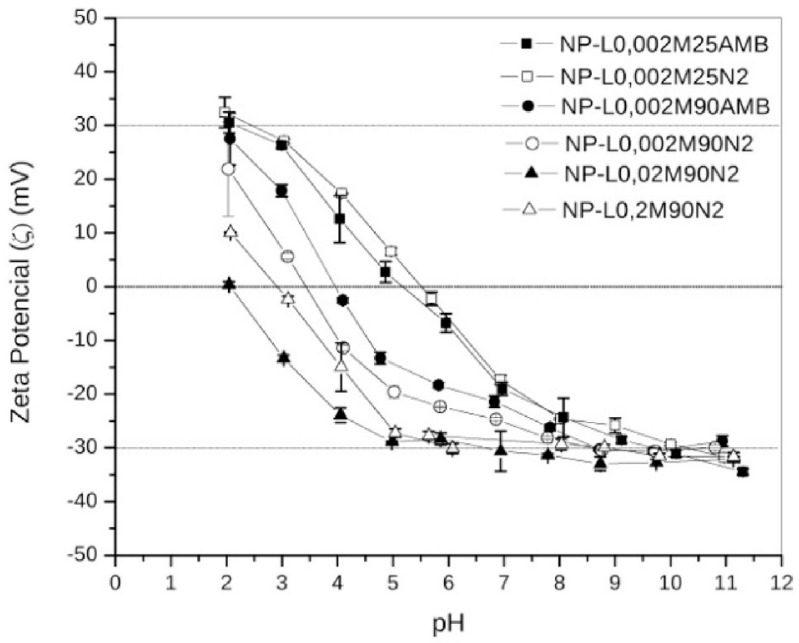

(b)

Fig. 7. Zeta potential measurements as a function of $\mathrm{pH}$ for (a) $\mathrm{Fe}_{3} \mathrm{O}_{4}$ nanoparticles and (b) EDTA-functionalized $\mathrm{Fe}_{3} \mathrm{O}_{4}$ nanoparticles. 
indicates that the EDTA binds on the surface of nanoparticles at these sites $[11,12,14]$.

The FTIR results showed that occurred the EDTA functionalization in all samples of magnetite. Already Yang et al. [13] synthesized the $\mathrm{Fe}_{3} \mathrm{O}_{4}$-EDTA nanoparticles by the coprecipitation method keeping the product at $90{ }^{\circ} \mathrm{C}$ for $30 \mathrm{~min}$. The EDTA functionalization shows that the EDTA exhibits high chemical affinity for $\mathrm{Fe}_{3} \mathrm{O}_{4}$ nanoparticles, even at room temperature. Moreover, the EDTA addition should be a cheap and satisfactory alternative for protection and functionalization of nanostructures. This system shows high potential to metal [12] and anions [13] adsorption. The FTIR spectra also show that the interaction between EDTA and magnetite occurs by the carboxylate anion. This result enables future functionalizations, since there is another carboxylate group in addition to tertiary nitrogens.

Fig. 7 shows the zeta potential curves $(\zeta)$ as a function of $\mathrm{pH}$ for the $\mathrm{Fe}_{3} \mathrm{O}_{4}$ nanoparticles (Fig. 7a) and for the $\mathrm{Fe}_{3} \mathrm{O}_{4}$-EDTA nanoparticles (Fig. 7b). In the case of the naked $\mathrm{Fe}_{3} \mathrm{O}_{4}$ nanoparticles, the zeta potential was found to be more influenced by the synthesis temperature than by atmospheric conditions. This result is reflected in the graph showing crystallite size as evaluated by the Scherrer equation (Fig. 2), in which the nanostructures exposed to the higher temperature $\left(90{ }^{\circ} \mathrm{C}\right.$ in the nitrogen atmosphere) increased slightly in size. This increase led to greater colloidal instability in these samples, and changes in the isoelectric point to lower $\mathrm{pH}$ values were found in the samples synthesized at $90^{\circ} \mathrm{C}$. In the case of the EDTA-functionalized $\mathrm{Fe}_{3} \mathrm{O}_{4}$ nanoparticles, behavior similar to that which was observed in the $\mathrm{Fe}_{3} \mathrm{O}_{4}$ nanoparticles was seen in the samples synthesized at $25{ }^{\circ} \mathrm{C}$. Increased isoelectric points were found among the samples synthesized at $90{ }^{\circ} \mathrm{C}$.

These results show that the charge density associated with the EDTA carboxyl group is predominant, and they are consistent with the results indicated by the IR spectroscopy. The analysis involving the concentration of EDTA (NP-L0.2M90N2 and NP-L0.02M90N2) found low isoelectric points due to high surface energy caused by the reduction in size, which changed the distribution of charges on the surface of the nanoparticles. These results are very promising for environmental remediation applications in the adsorption of heavy metals on the nanoparticles surface.

\section{Conclusions}

The results show that, in synthesis, the inert atmosphere associated with the co-precipitation method is fundamental for preventing the oxidation of magnetite. Furthermore, the X-ray diffraction analyses determined that temperature influences crystalline size, since the growth stage is favored at higher temperatures. The results reflect high homogeneity in nanoparticle shape and size in the samples studied. The addition of EDTA was also found to act as a barrier to the growth of nanoparticle nuclei, thus decreasing nanoparticle size. The functionalization of the surface of magnetite was confirmed by the Fourier transform infrared (FTIR) spectroscopy analyses and the development of new bands associated with EDTA. The zeta potential measurements showed that the distribution of charges on the surface of the samples is affected by synthesis temperatures and EDTA concentrations.

\section{Acknowledgements}

The authors are grateful for the support from the Institutional Scientific Research Grant Program of São Paulo State University (PROPe/ UNESP) - First Project Grant Request No. 775.

\section{References}

[1] A.K. Gupta, M. Gupta, Synthesis and surface engineering of iron nanoparticles for biomedical applications, Biomaterials 26 (2005) 3995-4021.

[2] J. Yang, S.B. Park, H.G. Yooh, Y.M. Huh, S. Haam, Preparation of poly $\varepsilon$-caprolactone nanoparticles containing magnetite for magnetic drug carrier, Int. J. Pharm. 324 (2006) 185-190.

[3] Z. Wang, R. Qiao, Na Tanga, Z. Lud, H. Wanga, Z. Zhange, X. Xuef, Z. Huangf, S. Zhangf, G. Zhanga, Y. Lif, Active targeting theranostic iron oxide nanoparticles for MRI and magnetic resonance-guided focused ultrasound ablation of lung cancer, Biomaterials 127 (2017) 25-35.

[4] G.S. Parkinson, Iron oxide surfaces, Surf. Sci. Rep. 71 (2016) 272-365.

[5] A. Akbarzadeh, M. Samiei, S. Davaran, Magnetic nanoparticles: preparation, physical properties, and applications in biomedicine, Nanoscale Res. Lett. 7 (2012) $144-157$.

[6] R.M. Comell, U. Schwertmann, The Iron Oxides - Structure, Properties, Reactions,

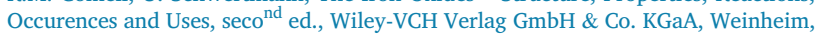
2003.

[7] S.C.N. Tang, I.M.C. Lo, Magnetic nanoparticle: essential factors for sustainable environmental applications, Water Res. 47 (2013) 2613-2632.

[8] R. Bini, R.F.C. Marques, F.J. Santos, J.A. Chaker, M. Jafelicci Jr., Synthesis and functionalization of magnetite nanoparticles with different alkoxysilanes, J. Magn. Magn. Mater 324 (2012) 534-539.

[9] An-Hui Lu, E.L. Salabas, F.S. Schüth, Magnetic nanoparticles: syntesis, protection, functionalization, and application, Angew. Chem. Int. Ed. 46 (2007) 1222-1244.

[10] S. Laurent, D. Forge, M. Port, et al., Magnetic iron oxide nanoparticles: synthesis stabilization, Vvectorization, physicochemical characterizations, and biological applications, Chem. Rev. 108 (2008) 2064-2110.

[11] R.J. Hunter, Introduction to Modern Colloid Science, Oxford University Press, New York, 1993.

[12] C.L. Warner, R.S. Addleman, A.D. Cinson, et al., High-Performance, superparamagnetic, nanoparticle-based heavy metals sorbents for removal of contaminants from natural Waters, ChemSusChem 3 (2010) 749-757.

[13] J. Yang, Q. Zeng, L. Peng, et al., La-EDTA coated $\mathrm{Fe}_{3} \mathrm{O}_{4}$ nanomaterial: preparation and application in removal of fosfate from water, J. Environ. Sci. 25 (2013) 413-418.

[14] K. Azizi, E. Ghonchepour, M. Karimi, A. Heydari, Encapsulation of Pd (II) into superparamagnetic nanoparticles grafted with EDTA and their catalytic activity towards reduction of nitroarenes and Suzuki-Miyaura coupling, Appl. Organomet. Chem. 29 (2015) 187-194.

[15] B.D. Cullity, Elements of X-Ray Diffraction. 2nd ED., London, 1977, p. 411.

[16] P.E. Tomaszewski, The uncertainly in grain size calculation from X-ray diffraction data, Phase Transitions 83 (2013) 260-266.

[17] M.A. Martins, T. Trindade, Os nanomateriais e a descoberta de novos mundos na bancada do químico, Quim. Nova 35 (2012) 1434-1446. 